\title{
Remote sensing and GIS based ecological modelling of potential red deer habitats in the test site region DEMMIN (TERENO)
}

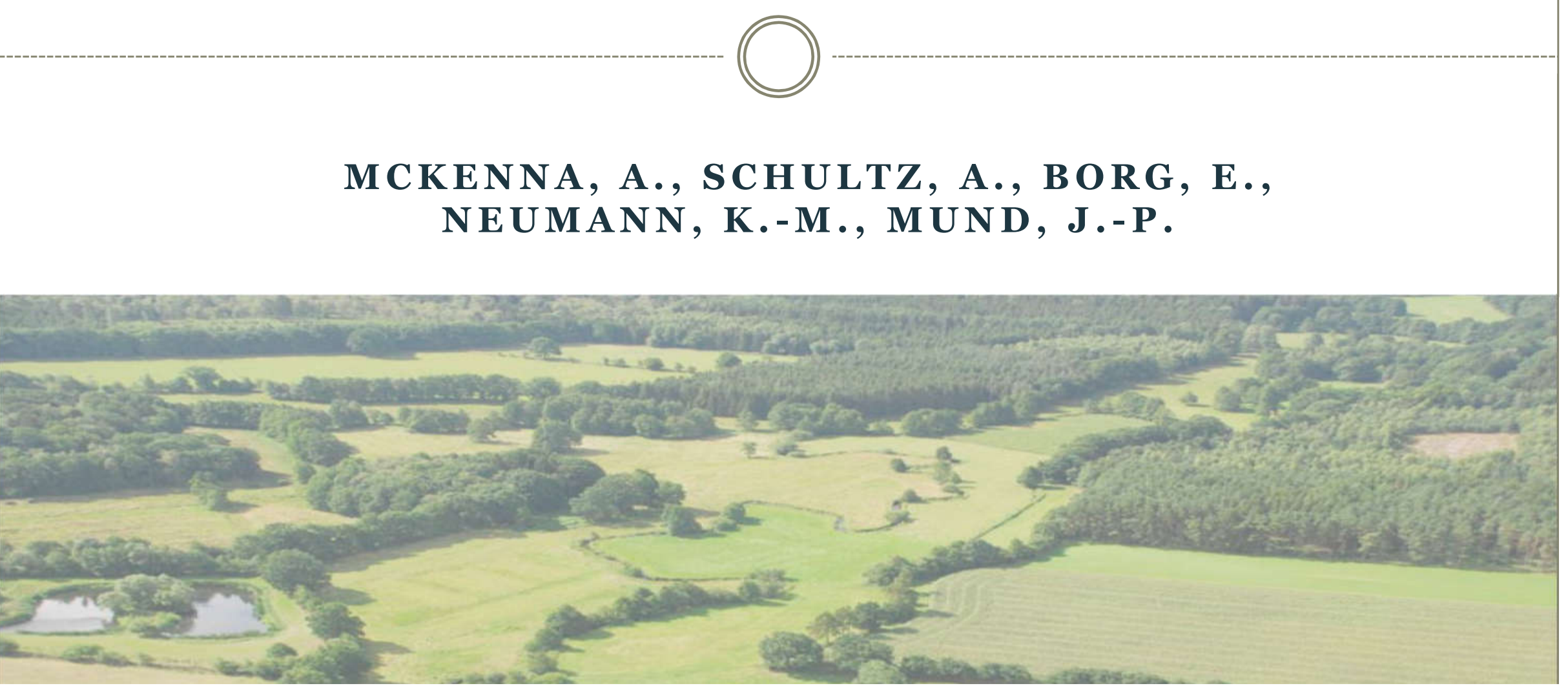




\section{Background \\ Material and Methods \\ Processing of data \\ Statistical analysis}

Results

Discussion

Literature

\section{Background}

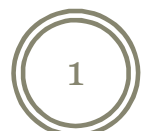

- The TERENO Initiative aims to study climate and land use changes as well as their regional impacts in order to formulate a sustainable socio-economic framework and estimate its ecological consequences (e.g. Heinrich et al., 2018, Bogena et al., 2019).

- In both cases, the strategic aim is to optimize the sustainable use and extraction of natural resources in order to protect natural capacities. In particular, the conservation and enhancement of biodiversity requires increasing attention.

- The above-mentioned land use change is more or less pronounced on different spatial and temporal scales (e.g. Schönwiese, 2008; Büntgen et al., 2013).

- The scientific community responded to the challenges by developing experiments and monitoring platforms (e.g. Osmond et al., 2004; Knorr et al., 2005, Mollenhauer, 2018).

- Recording biodiversity at regional and superregional levels under changing environmental conditions requires comprehensive monitoring based on sound concepts. Yet, the provision of extensive data sets, required for such monitoring, is cost and resource intensive.

- In the absence of specific quantitative datasets it is necessary to use every suitable and available data source to create as full a picture as possible of the complex interactions within ecosystems. In the following study, we will use an example to show how conclusions about the distribution of large mammals in a landscape dominated by humans can be derived from spatially and temporally uncertain data. 


\begin{tabular}{|c|c|}
\hline $\begin{array}{c}\text { Background } \\
\text { Material and } \\
\text { Methods }\end{array}$ & Objectives \\
$\begin{array}{c}\text { Processing of } \\
\text { data }\end{array}$ & The main objectives of the project are: \\
$\begin{array}{c}\text { Statistical } \\
\text { analysis }\end{array}$ & $\begin{array}{l}\text { Target-oriented pre-processing of uncertain and incomplete spatial } \\
\text { ecological observation data (hunting data reports) and } \\
\text { identification of correlated landscape structure metrics based on } \\
\text { precise RS data (Global Forest Change) }\end{array}$ \\
Results & $\begin{array}{l}\text { Development of a binary classifier for the prediction of habitat } \\
\text { suitability of Red Deer }\end{array}$ \\
Discussion & $\begin{array}{l}\text { Implementation and evaluation of the derived binary classifier } \\
\text { within the test site region DEMMIN (TERENO) }\end{array}$ \\
\hline Literature & \\
\hline
\end{tabular}




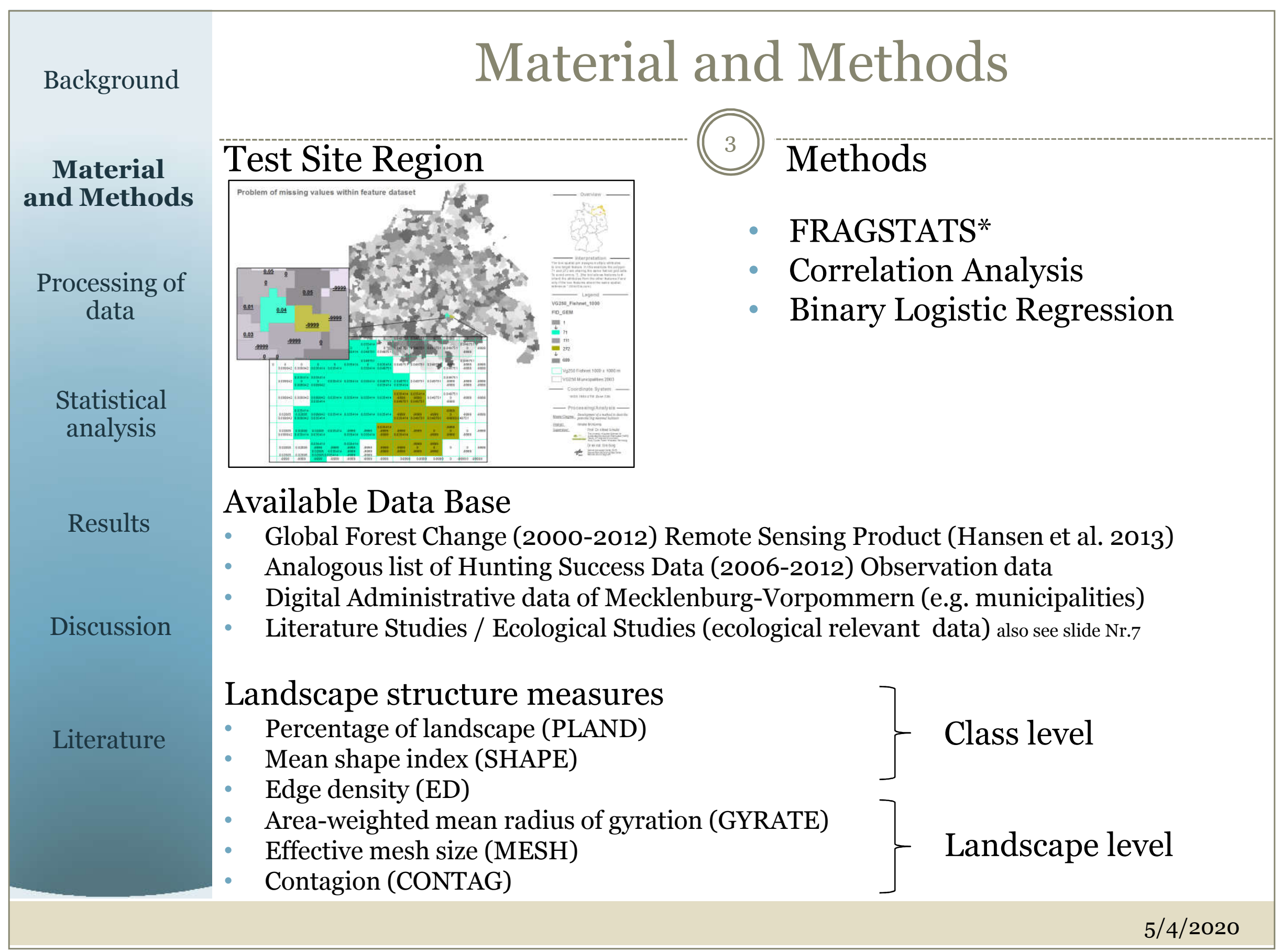




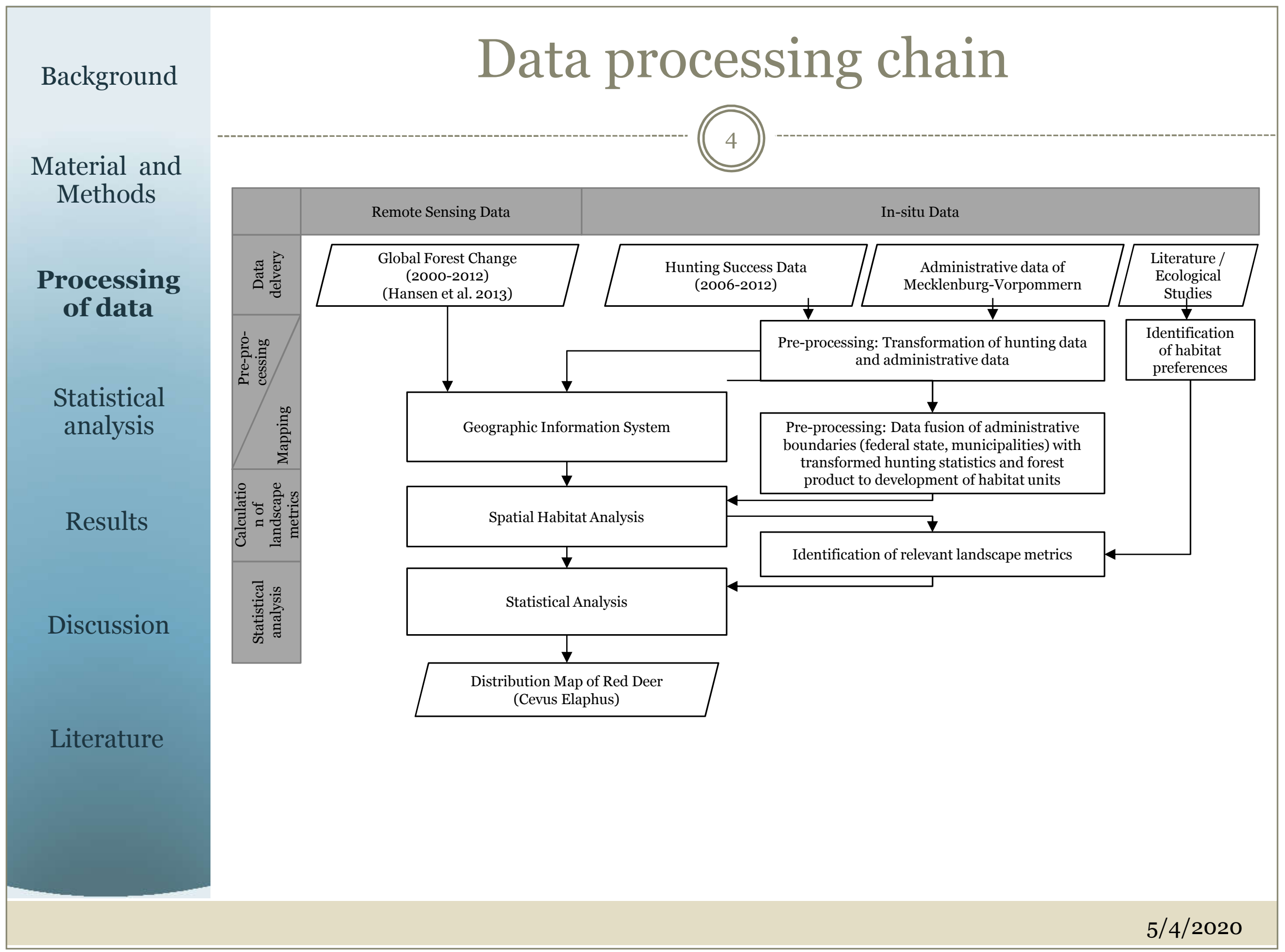




\begin{tabular}{|c|c|}
\hline Background & Binary classifier \\
\hline Material and & Hypothesis \\
\hline $\begin{array}{c}\text { Processing of } \\
\text { data }\end{array}$ & $\begin{array}{l}\text { It is supposed that Red Deer habitat suitability is dependent on } \\
\text { landscape structure and can be appropriately expressed with } \\
\text { landscape structure metrics: }\end{array}$ \\
\hline Statistical & $\begin{array}{l}\text { Binary Logistic Regression is used as classifier (advantage: no } \\
\text { particular data assumptions have to be fulfilled) }\end{array}$ \\
\hline Results & $\begin{array}{l}\text { area-wide classified habitats are divided in less or more preferred } \\
\text { habitats based on statistical reasoning (habitat suitability o or 1) } \\
\text { and serve as dichotomous dependent variable }\end{array}$ \\
\hline Diccuscion & $\begin{array}{l}\text { landscape structure metrics based on RS data serve as independent } \\
\text { variables }\end{array}$ \\
\hline Literature & $\begin{array}{l}\text { the derived regression function with unstandardized coefficients } \\
\text { serves as binary classifier; standardized coefficients (King, 2007) } \\
\text { allow comparative evaluation of input variables (that is ecological } \\
\text { importance of metrics) }\end{array}$ \\
\hline & $5 / 4 / 2020$ \\
\hline
\end{tabular}


Folie 6 und 7 zusammenfassen!

Alfred schickt mir Inhalt zur statistik Folie!

McKenna, Amelie; 01.05.2020 


\begin{tabular}{|c|c|c|c|c|c|c|c|c|}
\hline \multirow{2}{*}{\multicolumn{9}{|c|}{ Background }} \\
\hline & & & & & & & & \\
\hline & \multicolumn{3}{|c|}{ Summary of output variables } & & -andscape metrics & Uns & ression & $\begin{array}{c}\text { Standardized } \\
\text { regression coefficient }\end{array}$ \\
\hline \multirow{3}{*}{$\begin{array}{c}\text { Processing of } \\
\text { data }\end{array}$} & \multirow[t]{2}{*}{ Nagelkerke $R^{2}$} & \multirow{2}{*}{\multicolumn{2}{|c|}{ Overall Classification }} & Cont & agion & & -0.027 & -0.166 \\
\hline & & & & Edge & density & & -0.092 & -0.065 \\
\hline & 0.199 & \multicolumn{2}{|r|}{68.8} & Perce & entage of land & & 0.026 & 0.164 \\
\hline \multirow{2}{*}{$\begin{array}{l}\text { Statistical } \\
\text { analysis }\end{array}$} & & \multicolumn{5}{|c|}{ Habitat Model - Final } & & \\
\hline & & Landscape metrics & B & Wald statistic & \begin{tabular}{c|c|} 
p-value of Wald \\
chi-square \\
statistics
\end{tabular} & $\operatorname{Exp}(B)$ & & \\
\hline \multirow{4}{*}{ Discussion } & & Contagion & -0.027 & & 0.000 & 0.974 & & \\
\hline & & \begin{tabular}{|l} 
Edge density \\
\end{tabular} & -0.092 & 37.445 & 0.000 & 0.912 & & \\
\hline & & \begin{tabular}{|l|} 
Percentage of \\
landscape
\end{tabular} & 0.026 & 379.739 & 0.000 & 1.027 & & \\
\hline & & Constant & 0.719 & 13.620 & 0.000 & 2.052 & & \\
\hline \multirow{4}{*}{ Literature } & \multirow{2}{*}{\multicolumn{8}{|c|}{$+e$}} \\
\hline & & & & & & & & \\
\hline & $\begin{array}{l}\mathrm{P}(\mathrm{HIS}=1) \\
b_{o}, b_{1}, b_{2}, b_{3}\end{array}$ & \multicolumn{7}{|c|}{$\begin{array}{l}=\text { predicts whether habitats are more or less preferred } \\
=\text { coefficient (or weight) }\end{array}$} \\
\hline & & & & & & & & $5 / 4 / 2020$ \\
\hline
\end{tabular}




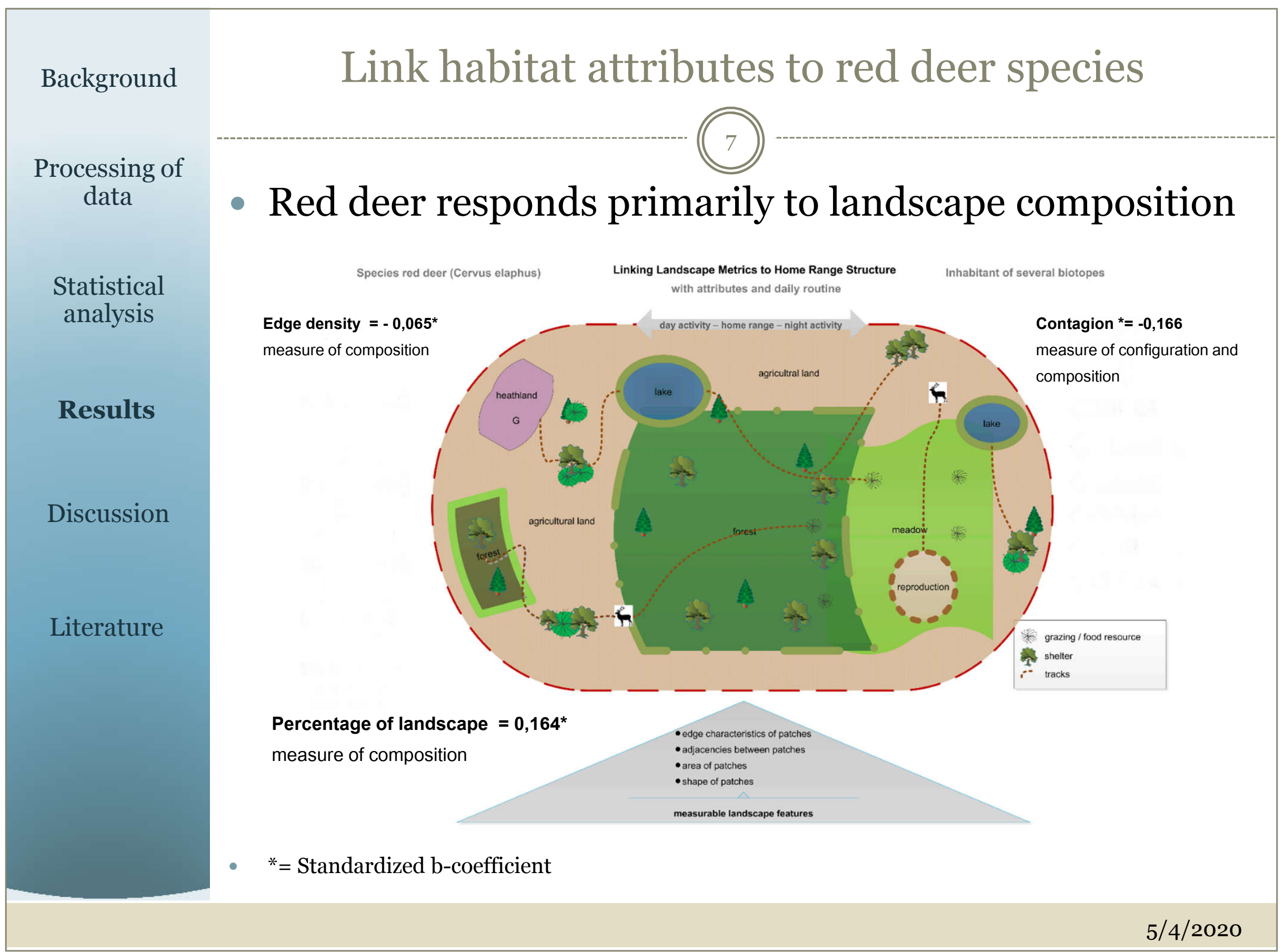




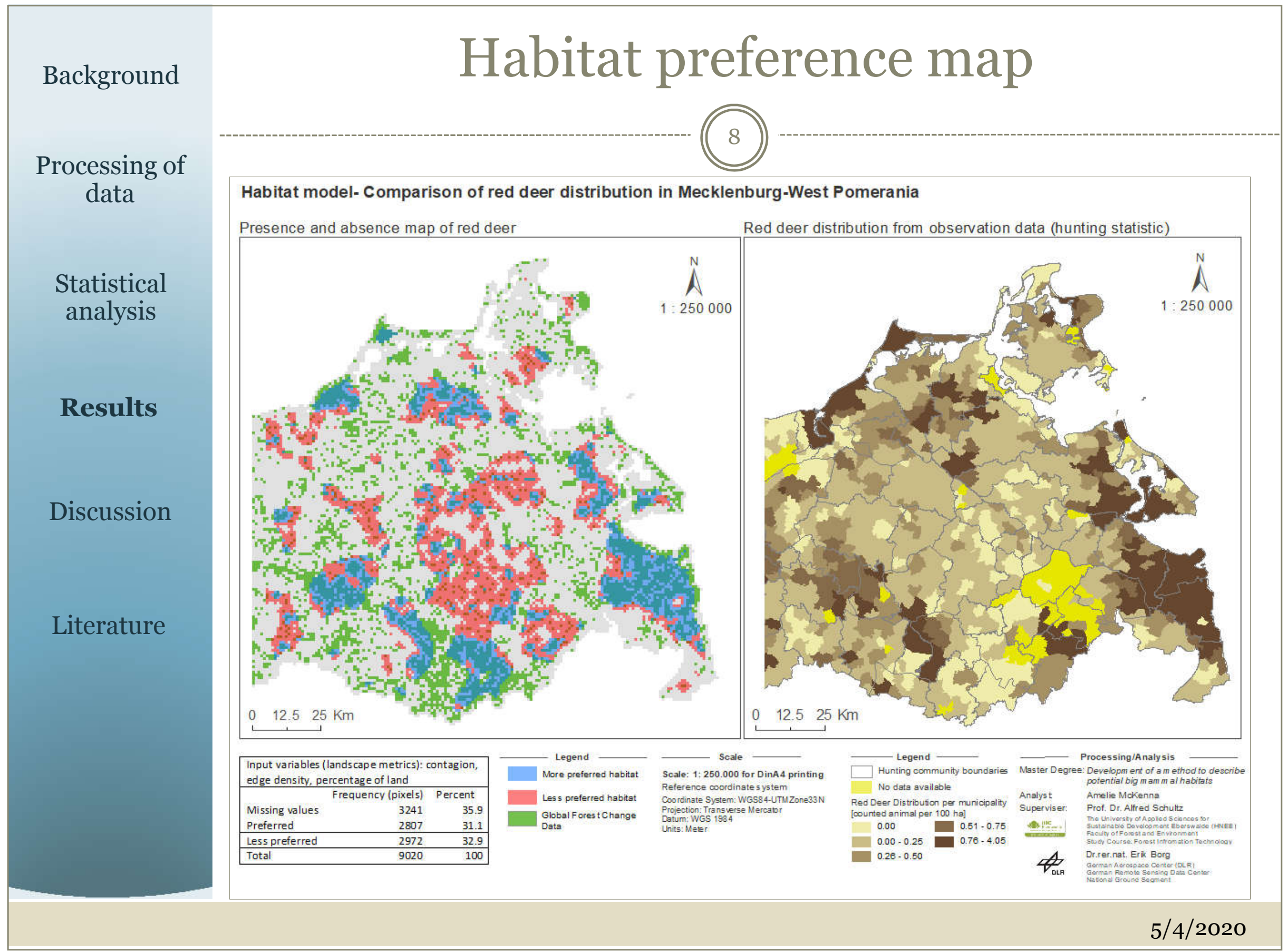




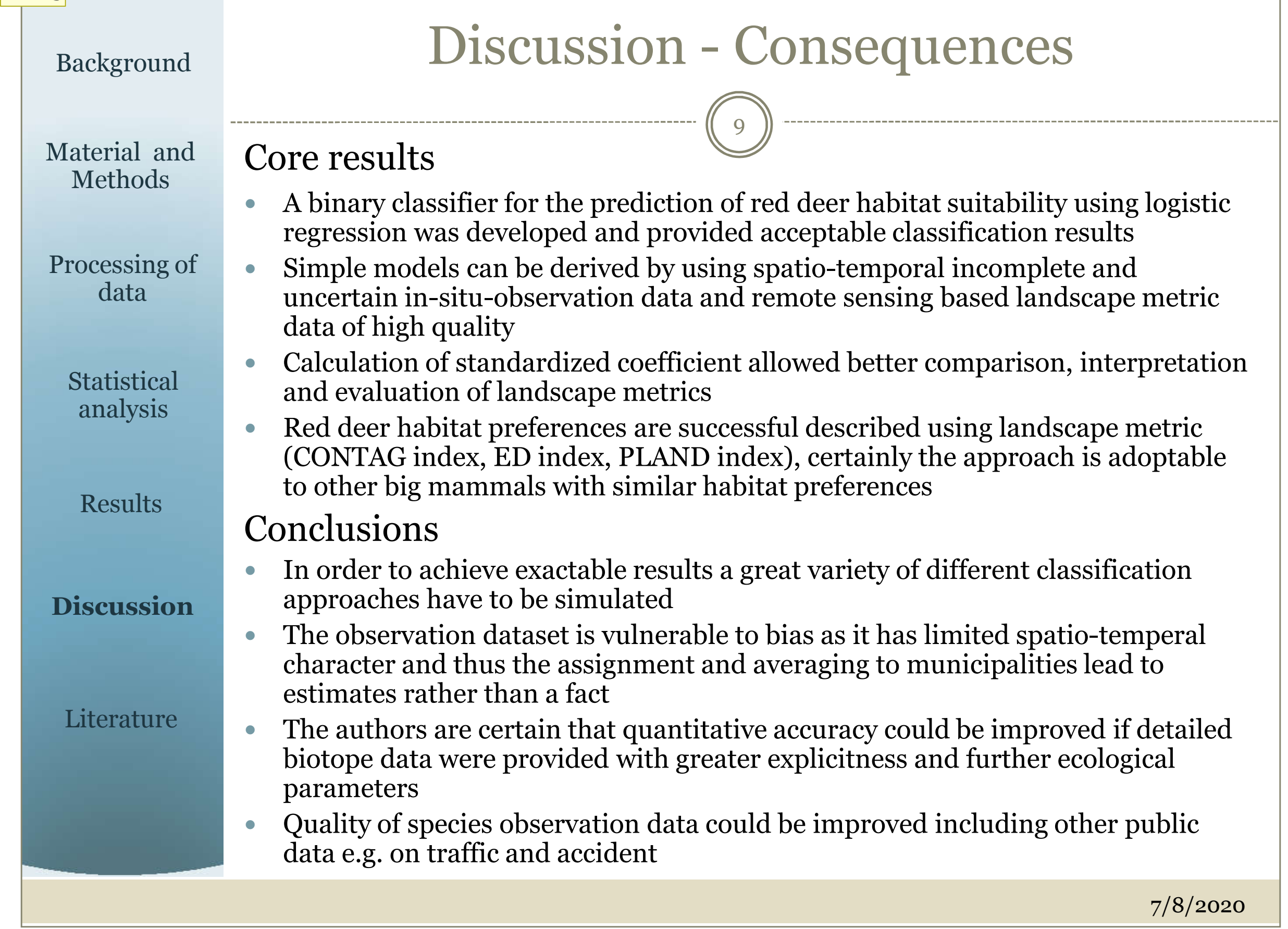


Folie 10

MA15

Bitte um Überprüfung der folie

McKenna, Amelie; 04.05.2020 


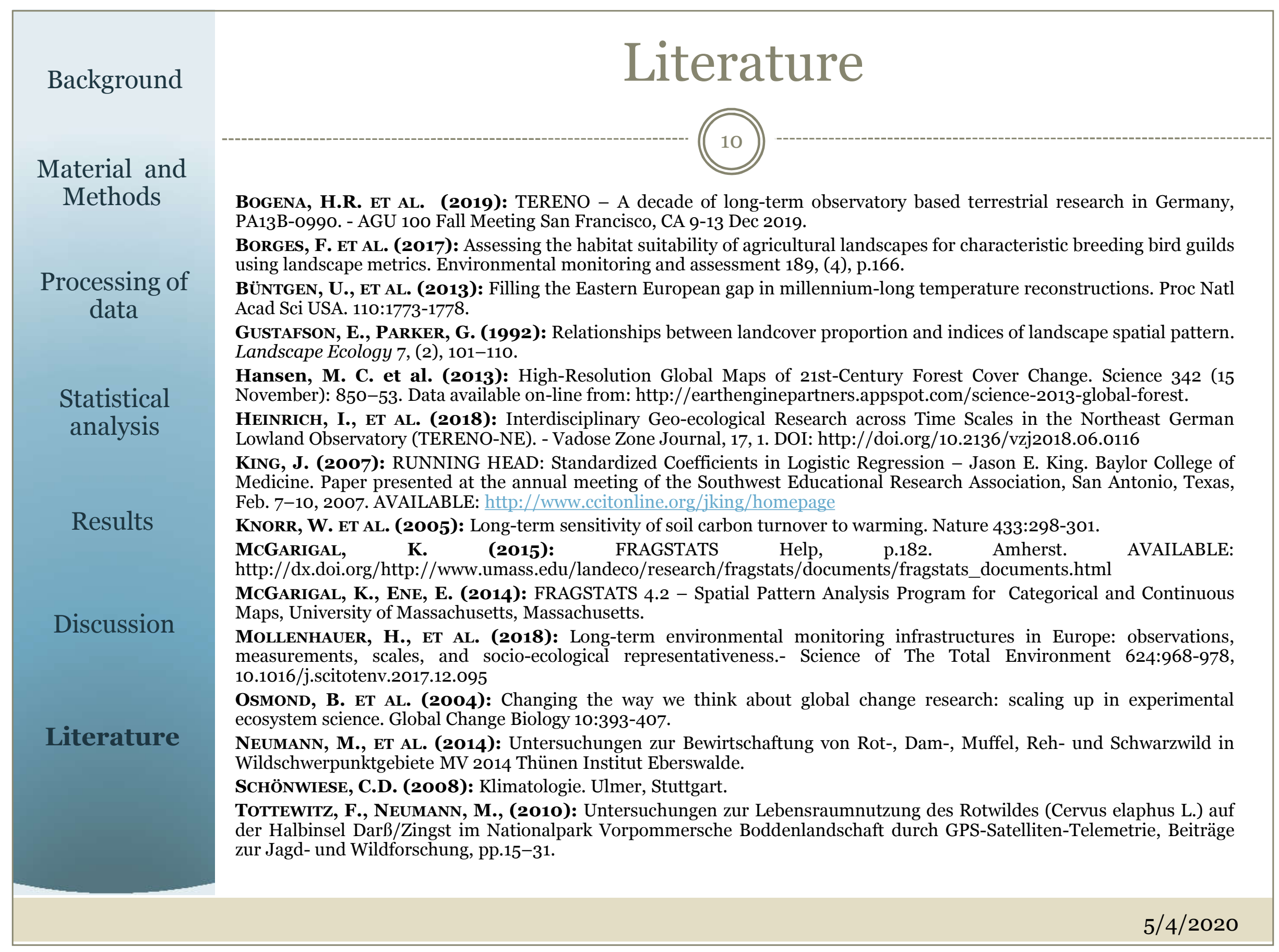




\section{Thank you for your attention!}

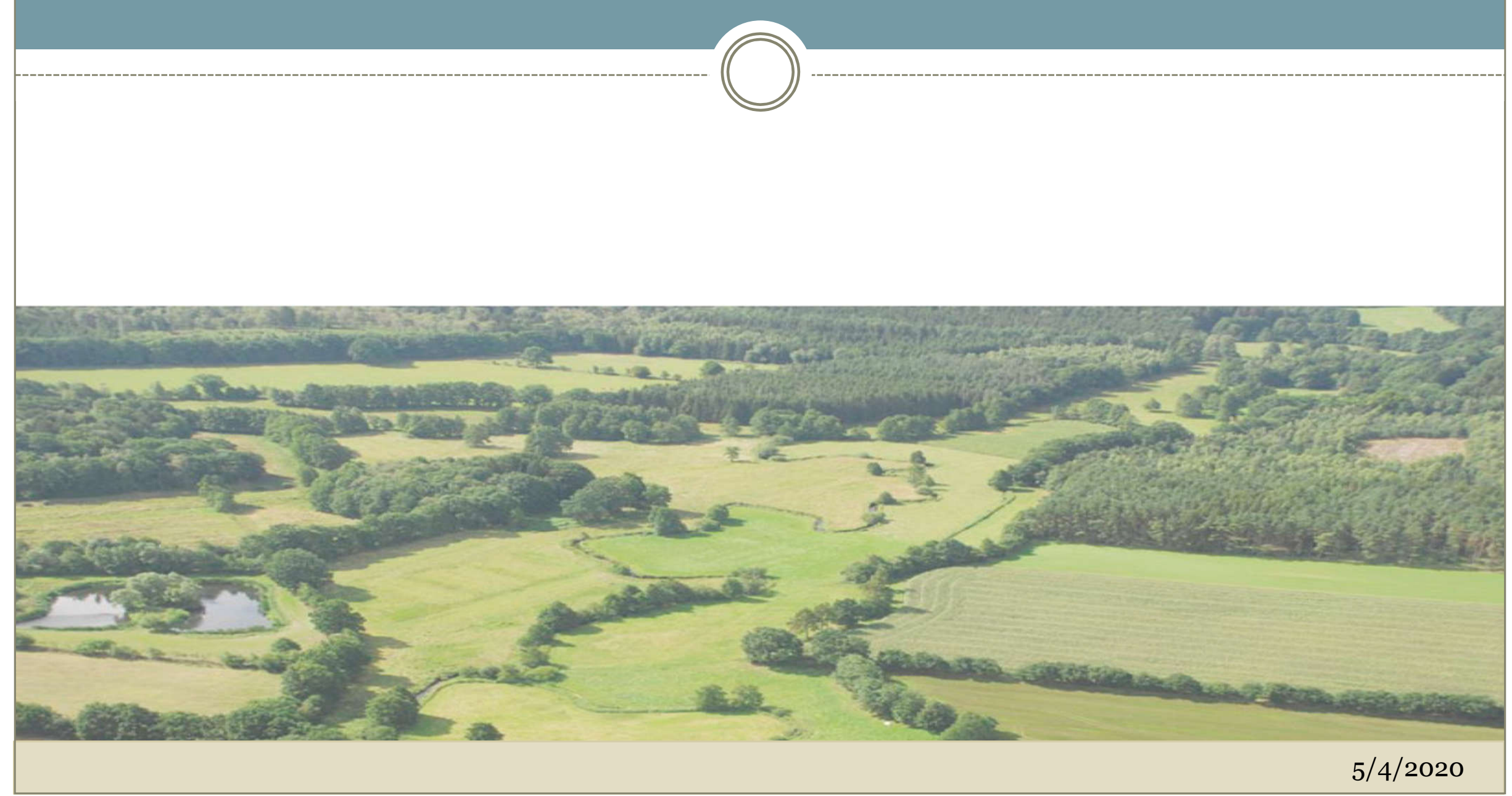

